\title{
BIBLIOTECONOMIA APLICADA AO CUIDADO EM SAÚDE: EXPERIÊNCIA DOCENTE
}

\author{
LIBRARIANSHIP APPLIED TO HEALTH CARE: \\ TEACHING EXPERIENCE
}

\author{
Amanda Damasceno de Souza \\ Maurício Barcellos Almeidab
}

\begin{abstract}
RESUMO
Introdução: A atuação do bibliotecário em Medicina e áreas correlatas é uma realidade. A Biblioteca Hospitalar, por exemplo, é um campo de atuação promissor. Entretanto, ainda é necessário inserir o ensino da Biblioteconomia Clínica na graduação com disciplinas cujo enfoque seja a informação em saúde e as atividades pertinentes à Biblioteca Hospitalar. Objetivo: $O$ presente artigo tem como objetivo descrever experiência em docência universitária no curso de Biblioteconomia, de forma a fomentar a inserção desse tipo de especialidade nos currículos de graduação. Metodologia: Descreve-se as etapas da experiência vivenciada por uma discente strictu senso no período de estágio docente em um Programa de Pós-Graduação da Ciência da Informação. A disciplina ministrada denominou-se "Biblioteconomia aplicada ao cuidado em Saúde", e objetivava proporcionar a complementação do conhecimento na área de informação em saúde, possibilitando a atualização em temas relacionados a organização e recuperação da informação em saúde. Resultados: O relato descreveu as teorias ministradas em sala de aulas, as atividades práticas, avaliativas, visita técnica e palestras, bem como o planejamento de todo o arcabouço teórico-prático necessário. Conclusões: Como experiência docente, a disciplina possibilitou a vivência em todas as etapas primordiais na formação pedagógica; como experiência para os discentes de graduação, ampliou a possibilidade de atuação em campos promissores da como as Bibliotecas Hospitalares. Conclui-se que é essencial que a graduação em Biblioteconomia contemple disciplinas as quais os graduandos possam desenvolver competências para atender as necessidades de informação em saúde, as quais são muitas em tempos recentes.
\end{abstract}

Descritores: Docência. Estágio docente. Formação bibliotecária. Biblioteconomia Clínica. Biblioteca médica.

\footnotetext{
a Doutoranda em Gestão e Organização do Conhecimento pela Escola de Ciência da Informação da Universidade Federal de Minas Gerais (UFMG). E-mail: amandasd81@gmail.com.

b Doutor em Ciência da Informação pela Escola de Ciência da Informação da Universidade Federal de Minas Gerais (UFMG). Docente do Programa de Pós-Graduação em Gestão \& Organização do Conhecimento da Universidade Federal de Minas Gerais (UFMG). E-mail: mba@eci.ufmg.br.
} 


\section{INTRODUÇÃO}

Na década de 1970, a bibliotecária Gertrude Lamb, identificou a necessidade de interligar o conteúdo científico da Medicina com a prática clínica, de forma a utilizar o conhecimento disponível na assistência ao paciente. Nesse contexto, Lamb percebeu uma oportunidade para os bibliotecários, os quais poderiam realizar essa conexão entre o saber cientifico da Medicina e a prática clínica. Criou o primeiro programa Clinical Medical Librarian (CLM) na University of Missouri-Kansas para capacitar bibliotecários a trabalhar no âmbito hospitalar. Com o passar dos anos, os programas de Biblioteconomia Clínica se fortaleceram, reafirmando sua importância com o movimento da Medicina Baseada em Evidencia (MBE) e com o desenvolvimento das tecnologias para fontes de informação em saúde (LIPSCOMB, 2000). A Biblioteconomia Clínica foi descrita por Lipscomb (2000) como um dos conceitos mais inovadores na área de bibliotecas de Ciências da Vida. Lamb foi ainda a percussora em nomear o bibliotecário que atua em Biblioteca Hospitalar de Bibliotecário Clínico.

No Brasil, estudos de Crestana (2003) e Beraquet e Ciol (2009a) relatam experiências de atuação de bibliotecários em hospitais e a necessidade de ampliar o escopo dessa atuação para toda a área de saúde. Entretanto, ainda é necessário inserir devidamente o ensino da Biblioteconomia Clínica na graduação, com disciplinas que enfoquem informação em saúde e MBE.

Sob essa perspectiva, torna-se primordial ministrar uma disciplina que exibe essa alternativa e ensina aos discentes de Biblioteconomia e Arquivologia a como lidar com o domínio da informação no âmbito hospitalar. Nesse contexto, foi ofertada uma disciplina intitulada "Biblioteconomia aplicada aos cuidados com saúde" como estágio docente por uma doutoranda do Programa de PósGraduação na área de Gestão e Organização do Conhecimento. O presente artigo tem como objetivo relatar a experiência de planejamento, desenvolvimento e consecução de estágio em docência universitária junto aos alunos do curso de graduação em Biblioteconomia e Arquivologia da Escola de Ciência da Informação (ECI) da Universidade Federal de Minas Gerais (UFMG).

Como metodologia para a disciplina foram planejadas e abordadas 
temáticas relacionadas a informação em saúde por meio de aulas expositivas, realização de exercícios práticos, curso online, apresentação de palestras, visita técnica à arquivo de prontuários de pacientes, realização de seminário e uma avaliação final. Os conteúdos selecionados para a disciplina foram elaborados com base na experiência em Biblioteconomia Clínica da doutoranda, docente da disciplina, e pelo seu orientador do doutorado.

\section{O ENSINO E ELABORAÇÃO DA DISCIPLINA}

A disciplina foi ministrada no $1^{\circ}$ semestre de 2018 , ofertada como optativa, a alunos a partir do sexto período diurno da graduação. Tratava-se de disciplina com carga horaria de 60 horas, conduzida pelo Departamento de Teoria e Gestão da Informação da ECl.

O objetivo da disciplina foi proporcionar a complementação do conhecimento na área de informação em saúde, possibilitando a atualização e o aprofundamento em temas relacionados a organização e recuperação da informação em saúde. A proposta fornece ao aluno as habilidades necessárias, do ponto de vista teórico e prático, para atuação em Bibliotecas Hospitalares. Além disso, a disciplina debateu a experiência do bibliotecário que atua no hospital como importante mediador na tomada de decisão em saúde, no cenário brasileiro e mesmo internacional.

\subsection{Temáticas ABORDADAS}

O tema introdutório da disciplina foi a Biblioteconomia Clínica, seu surgimento e evolução nos Estados Unidos da América (EUA), e a atuação do bibliotecário em Bibliotecas Hospitalares. Para abordar esse tema, foi necessário contextualizar a MBE, na qual o bibliotecário tem a participação essencial ao formar o elo entre a busca da melhor evidência disponível para o tratamento e a situação clínica do paciente (FERNANDES; SOUZA, 2020). Sobre o Bibliotecário Clínico foram abordadas as habilidades necessárias segundo Beraquet e Ciol (2009b), a formação no Brasil e nos EUA, as associações de classe como a Medical Library Association (MLA), os eventos científicos de informação em 
saúde e principais periódicos especializados em temas de Biblioteconomia Clínica. Ao ensinar sobre o trabalho do bibliotecário em hospitais, um aspecto importante foi trazer para a sala de aula exemplos e casos reais de profissionais que atuavam nessa área, para que os alunos pudessem compreender quais habilidades e conhecimentos são necessários para trabalhar num campo dinâmico como a saúde.

Após introduzir a Biblioteconomia Clínica, foram abordadas as fontes de informação em saúde. Pellizzon, Población e Goldenberg (2003, p. 493), no contexto da Biblioteca Virtual em Saúde (BVS), conceituaram fonte de informação como "qualquer recurso que responda uma demanda por parte dos usuários, incluindo produtos e serviços de informação, pessoas ou rede de pessoas, programas de computador [...]". Nessa temática foram relacionadas as tipologias das fontes de informação da BVS, fontes gerais de informação em saúde, fontes especializadas e as fontes para suporte a MBE. Dentre as fontes para a MBE, o destaque foi para os chamados "sumários médicos", que publicam sínteses de tópicos das diversas especialidades médicas com informações relevantes para a tomada de decisão, incluindo o tratamento e a indicação do nível de evidência de estudos publicados. Entre as principais fontes de informação para suporte a MBE, destacam-se o Uptodate $\AA^{1}{ }^{1}$, Dynamed $\AA^{2}$, Cochrane $^{3}$ e The $B M{ }^{4}$. Também foram abordados os principais periódicos da Medicina, os estudos clínicos, as sociedades médicas, as fontes governamentais e, por fim, as Bases de Dados.

O terceiro tema abordado foi a pesquisa bibliográfica em saúde em bases de dados especializadas. As bases de dados em saúde ganham destaque por serem um instrumento essencial ao Bibliotecário Clínico, que precisa conhecer tanto as bases quanto o vocabulário controlado para realizar pesquisa bibliográfica. Com esse conhecimento, o Bibliotecário Clínico também pode ofertar cursos e treinamentos ao corpo clínico do hospital. Há uma diversidade de bases de dados pagas e com acesso aberto. No contexto brasileiro destaca-

\footnotetext{
1 https://www.uptodate.com/home

2 http://www.dynamed.com/home/

${ }^{3} \mathrm{https}: / /$ www.cochrane.org/

${ }^{4}$ British Medical Journal https://www.bmj.com/
} 
se a Bireme com a BVS 5 e no contexto internacional, e o PubMed ${ }^{6}$. Para pesquisas bibliográficas na Bireme é utilizado o vocabulário controlado Descritores em Ciências da Saúde (DeCS) e para pesquisas no PubMed é utilizado o Medical Subject Headings (MeSH). O MeSH é um vocabulário controlado, organizado hierarquicamente e produzido pela National Library of Medicine (NLM) para indexar artigos no PubMed (PUBMED, 2005).

Após as fontes de informação em saúde, o passo seguinte foi ensinar ao graduando como avaliar a qualidade das fontes de informação em saúde. A prática médica é complexa e não depende somente da informação e do conhecimento, mas também da capacidade de julgamento do médico e da sua habilidade de interagir com o paciente. Para isso, ao solicitar ao bibliotecário a informação para tomada de decisão, é primordial que o médico receba informação confiável de uma fonte adequada. Grande parte das informações disponibilizadas na Internet sobre doenças e tratamentos são inadequadas ou incompletas cientificamente. Lopes (2004) descreve estudos sobre a qualidade da informação em saúde na Web e relata os critérios de avaliação de instituições como a Health on the Net Foundation (HON). A HON é uma organização sem fins lucrativos, sediada em Genebra, que avalia a confiabilidade de informações em saúde na Web. Para isso criou um selo de certificação - o HONcode ${ }^{7}$ - para sinalizar aos usuários de informação em saúde que um determinado site da Internet foi previamente avaliado por critérios do HON.

A Biblioteca Hospitalar é o local onde a equipe médica busca por soluções de informação e também por suporte no desenvolvimento de pesquisas dentro da instituição. Para atender a essa demanda, faz-se necessário conhecer os aspectos concernentes a pesquisa clínica, ou seja, a pesquisa realizada com seres humanos ou que utilize dados de pacientes. Um hospital, em que seu corpo clínico realiza pesquisa clínica precisa de um Comitê de Ética em Pesquisa (CEP). A presença no CEP se faz necessária para avaliar todos os protocolos de pesquisa a serem desenvolvidos na instituição. O CEP é um colegiado

\footnotetext{
${ }^{5}$ http://www.bireme.br/php/index.php

${ }^{6} \mathrm{https}: / /$ www.ncbi.nlm.nih.gov/pubmed/

7 https://www.hon.ch/HONcode/
} 
interdisciplinar e independente que deve existir nas instituições que realizam pesquisas envolvendo seres humanos no Brasil. Sua missão é salvaguardar os direitos e a dignidade dos sujeitos das pesquisas e para a discussão do papel da pesquisa no desenvolvimento institucional e no desenvolvimento social da comunidade.

Assim, é importante que o bibliotecário clínico esteja preparado para atender as demandas por elaboração de projetos seguindo as diretrizes e normas regulamentadoras de pesquisas envolvendo seres humano descritos na resolução no 466 de 2012 (BRASIL, 2012), para auxiliar a equipe médica no desenvolvimento de pesquisas. Sobre funcionamento do CEP, a disciplina incluiu a legislação de ética em pesquisa no Brasil, com o objetivo de demonstrar a complexidade da informação que concerne ao paciente e a importância do bibliotecário em atuar nesse contexto. Aliada a parte teórica sobre ética em pesquisa, os alunos realizaram um curso online intitulado Introduction to Research Ethics, para compreensão do contexto da pesquisa envolvendo seres humanos em âmbito hospitalar.

O curso abordou a história da ética em pesquisa desde a Segunda Guerra Mundial, na qual atrocidades foram cometidas nos campos de concentração com a justificativa de pesquisa médica. Desde o final da guerra foram instituídas resoluções e condutas sobre boas práticas em pesquisa clínica para assegurar os direitos e proteção aos participantes de pesquisa. As pesquisas clínicas são estudos realizados com humanos para medir os parâmetros de segurança e eficácia de novos medicamentos, sendo essencial para assegurar a chegada de novas alternativas terapêuticas no mercado.

Ao abordar conteúdos essenciais à Bibliotecas Hospitalares como, por exemplo, o desenvolvimento de acervo em saúde, enfatizou-se a necessidade de informação no ambiente hospitalar aliada aos objetivos da instituição (BRASIL, 2009a). Para o desenvolvimento de acervo em saúde faz-se necessário conhecer os usuários da biblioteca e entender quais são suas necessidades de informação. Na disciplina em questão, o desenvolvimento de acervo em saúde foi demostrando por meio de exemplos práticos vivenciados pela doutoranda docente. Nessa temática foram abordados desde os usuários 
da informação em saúde, mais informações importantes para tomada de decisão em relação a diagnóstico, tratamento e prognóstico de enfermidades, a MBE, temas sobre a produção científica e pesquisa clínica na instituição hospitalar e até os critérios para seleção de acervo físico, virtual e descarte.

O último tema desenvolvido com os alunos foi concernente a gestão de documentos em saúde em arquivos de prontuários, bem como as resoluções do Conselho Federal de Medicina que regulamentam a organização, manuseio e guarda desses documentos. O prontuário é o documento utilizado para a assistência ao paciente e também é a principal fonte de informação para pesquisa clínica no âmbito hospitalar. A equipe em saúde utiliza a informação dos prontuários para questões administrativas, jurídicas e para fins de pesquisa. O Bibliotecário Clínico precisa saber auxiliar a equipe em saúde na produção científica utilizando os dados dos prontuários para isso. Ir além e contribuir com sua expertise na representação e recuperação da informação, buscando soluções para problemas de caráter semântico que envolvem a interoperabilidade de terminologias clínicas (GALVÃO; RICARTE, 2011).

\subsection{Planejamento e Desenvolvimento}

A disciplina foi planejada de forma que a teoria fosse ministrada em conjunto com atividades práticas de uma Biblioteca Hospitalar. A seguir são descritas as atividades desenvolvidas com os discentes em sala de aula.

\subsubsection{Atividades práticas}

As atividades práticas foram uma etapa importante da disciplina, para ensinar aos graduandos sobre serviços oferecidos por uma Biblioteca Hospitalar. As atividades foram:

a) Preenchimento de Currículo Lattes:

A Plataforma Lattes configura-se como a experiência do CNPq na integração de bases de dados de Currículos, de Grupos de pesquisa e de Instituições em um único Sistema de Informações. O Currículo Lattes é um registro da vida pregressa e atual dos estudantes e pesquisadores do país. $O$ 
preenchimento de Currículo Lattes é um serviço muito procurado na Biblioteca Hospitalar por residentes e por preceptores, para fins acadêmicos e de pesquisa clínica. Por esse motivo foi necessário que os graduandos tivessem experiência com esta plataforma, de forma que foi solicitado a cada aluno que preenchesse seu próprio currículo para fins de aprendizado.

b) Formatação nas normas de Vancouver:

A normalização bibliográfica é um componente chave da boa prática acadêmica e melhora a apresentação do trabalho científico. A formatação seguindo normas bibliográficas mostra que a sua escrita se baseia no conhecimento e informado pela leitura acadêmica apropriada, além de assegura que o leitor possa rastrear as fontes utilizadas no desenvolvimento do trabalho $e$ dar-Ihe crédito. Ao citar o trabalho ou as ideias de outra pessoa de forma adequada, evita-se o plágio (IMPERIAL COLLEGE LONDON, 2017).

$\mathrm{Na}$ graduação de Biblioteconomia os graduandos estudam a norma ABNT. Entretanto, muitos periódicos da área de saúde adotam o estilo ou normas de Vancouver. As normas de Vancouver são um conjunto de regras para a publicação de trabalhos no âmbito das ciências da saúde. Receberam esse nome devido a uma reunião que foi realizada na cidade de Vancouver, no Canadá, em 1978 que culminou na criação do Comitê Internacional de Editores de Revistas Médicas (ICMJE) 8 .

Para fins de aprendizado foi importante ensinar essa norma em sala de aula e, em seguida, foi solicitado que todos os trabalhos de pesquisa fossem formatados seguindo o padrão Vancouver.

c) Pesquisa em base de dados da saúde:

A prática da pesquisa bibliográfica em base de dados da saúde é uma das atividades mais importante da Biblioteca Hospitalar. Para treinar a realização da pesquisa com os alunos, foi necessário seguir cada etapa de um levantamento bibliográfico, desde a elaboração da questão de pesquisa, a escolha dos descritores em vocabulário controlado, a elaboração da estratégia com operadores booleanos, a realização da busca, a análise de assunto dos

\footnotetext{
${ }^{8}$ International Committee of Medical Journal Editors. Disponível em: http://www.icmje.org/.
} 
resultados e a formatação das referências selecionadas, nas normas de Vancouver.

d) Curso online do programa de educação sobre ética em pesquisa envolvendo seres humanos:

Dentro da temática CEP foi proposto aos alunos a participação em um curso sobre ética em pesquisa intitulado Introduction to Research Ethics. O curso encontra-se disponível na plataforma na Training and Resources in Research Ethics Evaluation (TRREE). A plataforma TRREE fornece acesso gratuito a um programa de ensino a distância e provê certificação internacional de ética em pesquisa na saúde envolvendo seres humanos. O curso é disponibilizado por módulos em vários idiomas incluindo Português. Aos graduandos foi solicitado a realização do módulo Introduction to Research Ethics. Para fins de avaliação, os alunos apresentaram o certificado de realização do curso.

e) Seminário:

Como metodologia de aprendizado das temáticas ministradas foi solicitado aos alunos a apresentação de um pôster eletrônico, no seminário ao final da disciplina. Alguns temas foram sugeridos aos alunos com base nos assuntos debatidos em sala de aula, ou temas propostos pelos próprios alunos sobre informação em saúde. Para elaboração do pôster foi solicitado para cada aluno a realização de uma pesquisa bibliográfica em base de dados utilizando 0 tema escolhido para o seminário.

\subsubsection{Palestras}

As palestras na formação dos graduandos são importantes para orientar discussões, compreender determinados assuntos, aprender com especialistas, dentre outras possibilidades. Para a disciplina foram propostas duas palestras, a primeira sobre "Avaliação de tecnologias em saúde e Medicina Baseada em Evidência: a atuação do bibliotecário clínico"9 e a segunda ao final do curso, intitulada "Introdução as Ontologias Biomédicas"10.

\footnotetext{
9 Proferida pela Bibliotecária Clínica "Mariana Ribeiro Fernandes". E-mail: nanaufmg@gmail.com

10 Proferida pela doutoranda do programa de Pós-Graduação em Gestão e Organização do Conhecimento “Jeanne Louize Emygdio”. E-mail: jeanne.emygdio@gmail.com.
} 
Avaliação de Tecnologias em Saúde (ATS) "é um processo de investigação das consequências clínicas, econômicas e sociais da utilização das tecnologias em saúde" (OPAS, 2020, p.1). As ATS são todo tipo de inovação em saúde como medicamentos, equipamentos e procedimentos técnicos, sistemas organizacionais, educacionais, de informação e de suporte, além dos programas e protocolos assistenciais, criados com objetivo de prestar assistência em saúde à população (BRASIL, 2009b). O bibliotecário tem atuação importante na ATS, ao realizar levantamento bibliográfico para suporte a elaboração de parecer técnico sobre a viabilidade em utilizar uma tecnologia em saúde. Esse tema foi introduzido por meio de palestra com uma bibliotecária atuante com ATS.

$\mathrm{Na}$ Ciência da Informação, as ontologias vêm sendo estudadas na representação do conhecimento (ALMEIDA, 2014) e no domínio da Medicina encontram um terreno fértil de pesquisa. Assim, esse tema foi abordado por meio de palestra na disciplina para que os alunos tivessem uma compreensão da temática ontologias biomédicas como soluções semânticas aplicáveis aos problemas terminológicos da Medicina.

\subsubsection{Visita Técnica}

Uma visita técnica tem papel fundamental para contribuir para a formação dos futuros profissionais ao mostrar aos alunos possíveis campos de atuação. Essa experiência promove a oportunidade de aprofundar os conhecimentos da ciência e o relacionar com aplicações tecnológicas. A visita técnica foi realizada no Arquivo de Prontuários de Pacientes da Santa Casa de Misericórdia de Belo Horizonte, especificamente na oncologia, com objetivo de auxiliar no processo de ensino-aprendizagem.

Para a visita técnica foi elaborado um roteiro para auxiliar os alunos a elaborar questões e aprender sobre atividades da instituição. $O$ roteiro era composto por tópicos sobre as regras de funcionamento, as atividades do arquivo, a finalidade do prontuário e sua guarda, as diferenças entre prontuário em papel e em formato eletrônico.

Por fim, na visita técnica foi abordado o prontuário como fonte de informação para o Registro Hospital de Câncer (RHC). Os RHC: 
são fontes sistemáticas de informações, instalados em hospitais gerais ou especializados em oncologia, com o objetivo de coletar dados referentes ao diagnóstico, tratamento e evolução dos casos de neoplasia maligna atendidos nessas instituições, sejam públicas, privadas, filantrópicas ou universitárias (INSTITUTO NACIONAL DE CÂNCER, 2010, p.17).

A finalidade era que os alunos pudessem conhecer o $\mathrm{RHC}$ de um hospital público de grande porte, além de demonstrar o Prontuário do Paciente como instrumento que vai além da documentação referente à assistência ao paciente. O prontuário, de fato, também funciona como fonte de informação em saúde para melhoria de processos administrativos, nas atividades de pesquisa e na vigilância epidemiológica do câncer.

\subsubsection{Avaliação}

As atividades avaliativas foram realizadas individualmente com a finalidade de que cada aluno tivesse a oportunidade de colocar em prática 0 conteúdo ministrado em sala de aula. Ao final da disciplina, os alunos fizeram uma prova que abordou todo conteúdo ministrado, incluindo a bibliografia recomendada (vide QUADRO 1).

Quadro 1 - Atividades avaliadas durantes a disciplina

\begin{tabular}{|l|l|l|}
\hline & \multicolumn{1}{|c|}{ Atividade } & \multicolumn{1}{c|}{ Nota } \\
\hline 1 & Elaboração de Currículo Lattes & 05 pontos \\
\hline 2 & Realização de curso online em sala de aula & 10 pontos \\
\hline 3 & Relatório da visita ao arquivo de prontuários do paciente & 10 pontos \\
\hline 4 & Exercício prático em base de dados da saúde & 25 pontos \\
\hline 5 & Seminário & 20 pontos \\
\hline 6 & Prova & 30 pontos \\
\hline & Total & $\mathbf{1 0 0}$ pontos \\
\hline
\end{tabular}

Fonte: Elaborada pelos autores para a ementa da disciplina.

\subsubsection{Bibliografia básica da disciplina}

A bibliografia básica foi composta por artigos seminais, no idioma português, dos principais conteúdos abordados (QUADRO 2). Para a bibliografia complementar foram sugeridas as principais fontes de informação em saúde disponíveis na Web, artigos seminais de Biblioteconomia Clínica e MBE no idioma inglês. 


\section{Quadro 2 - Bibliografia básica}

\begin{tabular}{|c|c|c|}
\hline Referência & $\begin{array}{c}\text { Ano de } \\
\text { publicação }\end{array}$ & Assunto \\
\hline $\begin{array}{l}\text { BERAQUET, V. S.M.; CIOL, R. Atuação do } \\
\text { bibliotecário em ambientes não tradicionais: } \\
\text { o campo da saúde. Tendências da } \\
\text { Pesquisa Brasileira em Ciência da } \\
\text { Informação, Brasília, v.3, n.1, p.127-137, } \\
\text { jan./dez. } 2010 \text {. }\end{array}$ & 2010 & $\begin{array}{l}\text { Bibliotecário } \\
\text { clínico }\end{array}$ \\
\hline $\begin{array}{l}\text { BERAQUET, V. S.; CIOL, R. O bibliotecário } \\
\text { clínico no Brasil: reflexões sobre uma } \\
\text { proposta de atuação em hospitais } \\
\text { universitários. DataGramaZero - Revista de } \\
\text { Ciência da Informação, v.10, n.2, abr. } 2009 .\end{array}$ & 2009 & $\begin{array}{l}\text { Bibliotecário } \\
\text { clínico }\end{array}$ \\
\hline $\begin{array}{l}\text { CIOL, R.; BERAQUET, V. S. M. O. Evidência } \\
\text { e informação: desafios da medicina para a } \\
\text { próxima década. Perspectivas em Ciência } \\
\text { da Informação, Belo Horizonte. v. 14, n. } 3 \text {, } \\
\text { p. } 221-230,2009 \text {. }\end{array}$ & 2009 & $\begin{array}{c}\text { Medicina } \\
\text { Baseada em } \\
\text { Evidência }\end{array}$ \\
\hline $\begin{array}{l}\text { FREITAS, F.; SCHULZ, S.; MORAES, E. } \\
\text { Pesquisa de terminologias e ontologias } \\
\text { atuais em biologia e medicina. RECIIS - R. } \\
\text { Eletr. de Com. Inf. Inov. Saúde. Rio de } \\
\text { Janeiro, v.3, n.1, p.8-20, mar., 2009. }\end{array}$ & 2009 & $\begin{array}{l}\text { Terminologias } \\
\text { clínicas }\end{array}$ \\
\hline $\begin{array}{l}\text { LOPES, I.L. Novos paradigmas para } \\
\text { avaliação da qualidade da informação em } \\
\text { saúde recuperada na Web. Ciência da } \\
\text { Informação, Brasília. v.33, n. 1, p. 81-90, } \\
\text { jan./ abr. 2004. }\end{array}$ & 2004 & $\begin{array}{l}\text { Avaliação de } \\
\text { fontes de } \\
\text { informação }\end{array}$ \\
\hline $\begin{array}{l}\text { CONSELHO FEDERAL DE MEDICINA. } \\
\text { Resolução CFM no } 1.638 \text { de 2002. Define } \\
\text { prontuário médico e torna obrigatória a } \\
\text { criação da Comissão de Revisão de } \\
\text { Prontuários nas instituições de saúde. Diário } \\
\text { Oficial da União. Brasilia. } 9 \text { agos 2002, } \\
\text { Seção I, p.184-5. }\end{array}$ & 2002 & Prontuários \\
\hline $\begin{array}{l}\text { CONSELHO FEDERAL DE MEDICINA. } \\
\text { Resolução CFM no } 1.821 \text { de } 2007 \text {. Aprova as } \\
\text { normas técnicas concernentes à digitalização } \\
\text { e uso dos sistemas informatizados para a } \\
\text { guarda e manuseio dos documentos dos } \\
\text { prontuários dos pacientes, autorizando a } \\
\text { eliminação do papel e a troca de informação } \\
\text { identificada em saúde. Diário Oficial da } \\
\text { União. Brasília. } 23 \text { nov. 2007, Seção I, p. } 252 \text {. }\end{array}$ & 2002 & $\begin{array}{l}\text { Prontuários } \\
\text { eletrônicos }\end{array}$ \\
\hline
\end{tabular}

Fonte: Elaborada pelos autores para a ementa da disciplina. 


\section{CONSIDERAÇÕES FINAIS}

A disciplina contou com a participação de mais de 10 graduandos do curso de Biblioteconomia. Embora também tenha sido ofertada para alunos do curso de arquivologia, não houve demanda por parte dos alunos desse curso. A participação dos alunos nas atividades foi satisfatória, fato demostrando pelo interesse em todos os temas abordados e pela excelência dos trabalhos apresentados no seminário final da disciplina.

Como experiência docente, a disciplina possibilitou a vivência das etapas importantes na formação pedagógica desde a elaboração da disciplina, da ementa, das aulas expositivas, de atividades práticas e avaliativas. A disciplina possibilitou a doutoranda desenvolver a prática didática e aos alunos ampliou a possibilidade de atuação em campos promissores da Biblioteconomia como as Bibliotecas Hospitalares.

O Bibliotecário Clínico executa o trabalho de assessoria ao corpo clínico do hospital, de forma que suas atividades envolvem a gestão da Biblioteca Hospitalar. Mas é preciso ir além: serviços para promover a produção científica, desenvolver acervo para viabilizar a MBE, auxiliar em questões éticas sobre informações concernentes a pesquisa com dados de pacientes, buscar soluções para a interoperabilidade entre terminologias clínicas, dentre outros, devem fazer parte dos encargos da Biblioteca Hospitalar. Para isso, é essencial que a graduação em Biblioteconomia contemple disciplinas através das quais os graduandos possam desenvolver competências para atender as necessidades de informação dos usuários da Biblioteca Hospitalar.

\section{REFERÊNCIAS}

ALMEIDA, M.B. Uma abordagem integrada sobre ontologias: Ciência da Informação, Ciência da Computação e Filosofia. Perspectivas em Ciência da Informação, Belo Horizonte, v. 19, n. 3, p. 242-258, set. 2014. Disponível em: http://portaldeperiodicos.eci.ufmg.br/index.php/pci/article/view/1736. Acesso em: 14 abr. 2020.

BRASIL. Ministério da Saúde. Secretaria-Executiva. Subsecretaria de Assuntos Administrativos.Política de Desenvolvimento de Coleções da Biblioteca do 
Ministério da Saúde .2. ed. Brasília : Editora do Ministério da Saúde, 2009a. 64 p. (Série A. Normas e Manuais Técnicos). Disponível em http://bvsms.saude.gov.br/bvs/publicacoes/politica_desenvolvimento_colecoes_ biblioteca_ms.pdf. Acesso em: 14 abr. 2020.

BRASIL. Ministério da Saúde. Secretaria-Executiva. Área de Economia da Saúde e Desenvolvimento. Avaliação de tecnologias em saúde: ferramentas para a gestão do SUS.Brasília: Editora do Ministério da Saúde, 2009b. 110 p. (Série A. Normas e Manuais Técnicos). Disponível em http://bvsms.saude.gov.br/bvs/publicacoes/avaliacao_tecnologias_saude_ferra mentas_gestao.pdf. Acesso em: 10 abr. 2020.

BRASIL. Ministério da Saúde. Conselho Nacional de Saúde. Resolução no 466, de 12 de dezembro de 2012. Dispõe sobre diretrizes e normas regulamentadoras de pesquisas envolvendo seres humanos. Diário Oficial [da] República Federativa do Brasil, Brasília, DF, 13 jun. 2013. Disponível em:

https://bvsms.saude.gov.br/bvs/saudelegis/cns/2013/res0466_12_12_2012.html Acesso em: 14 abr. 2020.

BERAQUET, V. S. M.; CIOL, R. Bases para o desenvolvimento da biblioteconomia clínica no hospital da PUC-Campinas: capacitação de bibliotecários. IN: Encontro Nacional de Pesquisa em Ciência da Informação, ENANCIB, X, GT 6 - Informação, Educação e Trabalho, 2009, João Pessoa, BR. Anais... 25 a 28 de outubro de 2009b. Disponível em http://enancib.ibict.br/index.php/enancib/xenancib/paper/viewFile/3279/2405. Acesso em: 14 abr. 2020.

BERAQUET, V. S. M.; CIOL, R. O bibliotecário clínico no brasil: reflexões sobre uma proposta de atuação em hospitais universitários. DataGramaZero, v. 10, n. 2, 2009b. Disponível em: <http://hdl.handle.net/20.500.11959/brapci/6464>. Acesso em: 10 abr. 2020.

FERNANDES, M. R.; SOUZA, A. D. Medicina baseada em evidência para tomada de decisão em serviços de saúde: o papel do bibliotecário clínico. Informação em Pauta, Fortaleza. v. 5, n. Especial, p. 36-51, 2020. Disponível em: http://hdl.handle.net/20.500.11959/brapci/136622. Acesso em: 10 abr. 2020.

CRESTANA, M.F. Bibliotecários da área médica: o discurso a respeito da profissão. Perspectivas em Ciência da Informação, Belo Horizonte, v. 8, n. 2, p.134-149, nov. 2003. Disponível em:

http://portaldeperiodicos.eci.ufmg.br/index.php/pci/article/view/365. Acesso em: 14 abr. 2020.

GALVAO, M. C.; RICARTE, I. L. O prontuário eletrônico do paciente no século XXI: contribuições necessárias da ciência da informação. InCID: Revista de Ciência da Informação e Documentação, v. 2, n. 2, p. 77-100, 9 dez. 2011. 
Disponível em: http://www.revistas.usp.br/incid/article/view/42353. Acesso em: 14 abr. 2020.

IMPERIAL COLLEGE LONDON.Citing \& Referencing: Vancouver Style. September 2017. Disponível em :https://www.imperial.ac.uk/media/imperialcollege/administration-and-support-services/library/public/vancouver.pdf.Acesso em: 14 abr. 2020.

INSTITUTO NACIONAL DE CÂNCER.Registros hospitalares de câncer: planejamento e gestão. 2 ed. Rio de Janeiro: INCA, 2010.536 p. Disponível em : https://www.inca.gov.br/sites/ufu.sti.inca.local/files//media/document//registroshospitalares-de-cancer-2010.pdf. Acesso em: 07 maio 2020.

LIPSCOMB, C.E.Clinical librarianship. Bulletin of the Medical Library Association, Chicago. v.88, n.4, p.393-5, 2000.

LOPES, I.L. Novos paradigmas para avaliação da qualidade da informação em saúde recuperada na Web. Ciência da Informação, v.33, n. 1, p. 81-90, jan./ abr. 2004.

\title{
ORGANIZAÇÃO PANAMERICANA DE SAÚDE (OPAS). Rede Brasileira de
} Avaliação de Tecnologias em Saúde disponibiliza estudos.2020.Disponível em:

https://www.paho.org/bra/index.php?option=com_content\&view=article\&id=318 4:rede-brasileira-de-avaliacao-de-tecnologias-em-saude-disponibilizaestudos\&ltemid=838. Acesso em: 10 abr. 2020.

PELLIZZON, R.F; POBLACIÓN, D.A.; GOLDENBERG, S. Pesquisa na área da saúde: seleção das principais fontes para acesso à literatura científica. Acta cirúrgica brasileira [serial online]. v.18, n.6,2003. Disponível em: http://www.scielo.br/acb. Acesso em: 10 abr. 2020.

PUBMED Help [Internet]. Bethesda (MD): National Center for Biotechnology Information (US), 2005. Disponível em:

https://www.ncbi.nlm.nih.gov/books/NBK3830/. Acesso em: 07 maio 2020.

\section{LIBRARIANSHIP APPLIED TO HEALTH CARE: TEACHING EXPERIENCE}

\begin{abstract}
Introduction: The role of librarians in Medicine and its fields is a reality. Acting in the Hospital Library, for example, is a promising field of action. However, it is still necessary to insert the proper teaching of Clinical Librarianship in undergraduate courses, focusing on health information and the relevant activities that take place within the Hospital Library. Objective: This article aims to describe the experience in teaching in the Library Science major to foster the insertion of this type of specialty in under graduation curricula. Methodology: We describe the stages of the experience experienced by a PhD candidate during the Teaching Internship required to by an Information Science
\end{abstract}


Graduate Program. The discipline taught was called "Librarianship Applied to Health Care" delivered with the aim of complementing the knowledge in the area of health information, updating topics about the organization and retrieval of health information. Results: The report describes as theories taught in class, the practical activities performed and evaluated, technical visits, lectures, as well as the planning of the required theoretical and practical framework. Conclusions: As a teaching experience, the discipline made it possible to experience all essential stages of the pedagogical training; as an experience for undergraduate students, it expanded the possibility of working in promising market fields as Hospital Libraries. We concluded that it is essential that the degree in Library and Information Science contemplates subjects such as we described, in which graduate students can obtain and use skills to meet health information requirements of Medical professionals.

Descriptors: Teaching. Teaching internship. Pedagogical formation. Clinical Librarian. Health Library.

\title{
BIBLIOTECONOMÍA APLICADA A LA ATENCIÓN MÉDICA: EXPERIENCIA DOCENTE
}

\begin{abstract}
RESUMEN
Introducción: El papel del bibliotecario en Medicina y áreas relacionadas es una realidad. La Biblioteca del Hospital, por ejemplo, es un campo de acción prometedor. Sin embargo, aún es necesario insertar la enseñanza de la Biblioteconomía Clínica en los cursos de pregrado con un enfoque en información de salud y actividades relevantes para la Biblioteca del Hospital. Objetivo: Este artículo tiene como objetivo describir la experiencia en la enseñanza universitaria en el curso de Bibliotecología, con el fin de fomentar la inserción de este tipo de especialidad en los planes de estudio de pregrado. Metodología: describe las etapas de la experiencia vivida por un estudiante estricto en el período de prácticas docentes en un Programa de Posgrado en Ciencias de la Información. La disciplina que se enseñó se denominó "Biblioteconomía aplicada a la atención de la salud" y tenía como objetivo proporcionar la complementación del conocimiento en el área de la información de salud, permitiendo la actualización de temas relacionados con la organización y la recuperación de información de salud. Resultados: El informe describió las teorías impartidas en el aula, las actividades prácticas, evaluativas, visitas técnicas y conferencias, así como la planificación de todo el marco teórico y práctico necesario. Conclusiones: como experiencia docente, la disciplina permitió experimentar todas las etapas esenciales de la formación pedagógica; Como una experiencia para estudiantes universitarios, amplió la posibilidad de actuar en campos prometedores como las bibliotecas hospitalarias. Se concluye que es esencial que el curso de pregrado en Biblioteconomía contemple materias en las que los estudiantes de pregrado puedan desarrollar competencias para satisfacer las necesidades de información de salud, que son muchas en los últimos tiempos.
\end{abstract}

Descriptores: Docencia. Prácticas docentes. Formación de bibliotecario. Biblioteconomía Clínica. Biblioteca médica.

Recebido em: 01.06.2020

Aceito em: 21.09.2020 